\title{
O enfermeiro gestor atuante no sistema carcerário: Habilidades, conhecimentos e atitudes necessárias para se fazer a humanização da assistência
}

The nurse manager active in the prison system: Skills, knowledge and attitudes necessary to do the humanization of care

El gerente de enfermería activo en el sistema penitenciario: Habilidades, conocimientos y actitudes necesarias para hacer la humanización de la atención

Layna Pereira de Amorim ORCID: https://orcid.org/0000-0002-9825-8848

Universidade Estácio de Sá, Brasil E-mail: enf.laynaamorim@outlook.com Lidiane Dias Reis ORCID: https://orcid.org/0000-0001-6577-6545 Universidade Estácio de Sá, Brasil E-mail: lidiane.dias@estacio.br Carla Miranda Pereira ORCID: https://orcid.org/0000-0002-5350-2229 Universidade Estácio de Sá, Brasil E-mail: carla_miranda26@hotmail.com

Caroline da Costa Pereira ORCID: https://orcid.org/0000-0002-1496-587X Universidade Estácio de Sá, Brasil E-mail: karol.hatha@gmail.com

Thays Chagas de Castro Rodrigues ORCID: https://orcid.org/0000-0003-4738-9095 Universidade Estácio de Sá, Brasil

E-mail: thays_chagas_castro@hotmail.com

\begin{abstract}
Resumo
Introdução: Sabemos que a assistência à saúde implementada no sistema prisional deve ser elaborada com o objetivo de prover a carência percebidas entre as Pessoas Privadas de Liberdade (PPL). Diante desse cenário, o enfermeiro deve desenvolver o cuidado como prática social oferecendo uma assistência integral que deve ser realizada de maneira humanizada, com a utilização de uma escuta qualificada, fazendo assim um cuidado de qualidade para a realização da assistência respeitando direitos e deveres éticos de sua profissão. Objetivo: Identificar as práticas assistenciais realizadas pela equipe de enfermagem para as PPL, conhecendo as habilidades, conhecimento e atitudes necessárias para se fazer a humanização da assistência do enfermeiro em um sistema prisional. Metodologia: Trata-se de um estudo com abordagem qualitativa, do tipo revisão integrativa, que teve como base de dados a Biblioteca Virtual de Saúde usando os Descritores em Ciências da Saúde (DeCS): saúde and enfermagem and prisões. Foram selecionados artigos completos, disponíveis, em língua portuguesa, inglesa e espanhola entre 2016 a 2021. Ao fim da aplicação dos critérios, emergiram 11 artigos para produção do conteúdo. Resultados: Foram observadas várias formas de se proporcionar o cuidado humanizado as PPL, destacando a valorização e reconhecimento dos profissionais de saúde e a articulação do mesmo ao promover saúde e acolhimento. Considerações Finais: A pesquisa levou a concluir que não basta pensar em humanização com enfoque apenas nessas pessoas, deve-se também enfatizar que os profissionais de saúde, por serem protagonistas do processo de cuidar, necessitam de reconhecimento e valorização do trabalho nesses setores.
\end{abstract}

Palavras-chave: Saúde; Enfermagem; Prisões.

\begin{abstract}
Introduction: We know that health care implemented in the prison system must be designed with the aim of providing the perceived shortages among Persons Deprived of Liberty (PPL). In view of this scenario, nurses must develop care as a social practice offering comprehensive care that must be performed in a humanized manner, with the use of qualified listening, thus providing quality care for the performance of care, respecting the ethical rights and duties of your profession. Objective: To identify the care practices performed by the nursing team for the PLP, knowing the skills, knowledge and attitudes necessary to make the humanization of nurses' care in a prison system. Methodology: This is a study with a qualitative approach, of the integrative review type, which had as a database the Virtual Health
\end{abstract}


Library using the Health Sciences Descriptors (DeCS): health and nursing and prisons. Complete articles were selected, available in Portuguese, English and Spanish between 2016 and 2021. At the end of the application of the criteria, 11 articles emerged for the production of the content. Results: Various ways of providing humanized care to the PLP were observed, highlighting the valorization and recognition of health professionals and their articulation when promoting health and welcoming. Final Considerations: The research led to the conclusion that it is not enough to think about humanization focusing only on these people, it must also be emphasized that health professionals, as they are protagonists of the care process, need recognition and appreciation of work in these sectors.

Keywords: Health; Nursing; Prisons.

\section{Resumen}

Introducción: Sabemos que la atención de salud implementada en el sistema penitenciario debe diseñarse con el objetivo de atender las carencias percibidas entre las Personas Privadas de Libertad (PPL). Diante desse cenário, o enfermeiro deve desenvolver o cuidado como prática social oferecendo uma assistência integral que deve ser realizada de maneira humanizada, com a utilização de uma escuta qualificada, fazendo assim um cuidado de qualidade para a realização da assistência respeitando direitos e deveres éticos de tu profesion. Objetivo: Identificar las prácticas asistenciales que realiza el equipo de enfermería para las PLP, conociendo las habilidades, conocimientos y actitudes necesarias para humanizar el cuidado de las enfermeras en un sistema penitenciario. Metodología: Se trata de un estudio con enfoque cualitativo, del tipo revisión integradora, que tuvo como base de datos la Biblioteca Virtual en Salud utilizando los Descriptores de Ciencias de la Salud (DeCS): salud y enfermería y prisiones. Se seleccionaron artículos completos, disponibles en portugués, inglés y español entre 2016 y 2021. Al final de la aplicación de los criterios, surgieron 11 artículos para la producción del contenido. Resultados: Se observaron diversas formas de brindar atención humanizada a las PLP, destacando la valorización y reconocimiento de los profesionales de la salud y su articulación a la hora de promover la salud y la acogida. Consideraciones finales: La investigación llevó a la conclusión de que no basta con pensar en la humanización concentrándose solo en estas personas, también se debe enfatizar que los profesionales de la salud, por ser protagonistas del proceso de atención, necesitan reconocimiento y valoración del trabajo en estos sectores.

Palabras clave: Salud; Enfermería; Cárceles.

\section{Introdução}

De acordo com os dados que o Ministério da Justiça informa, 520 mil pessoas encontram-se privadas de sua liberdade nos sistemas penitenciários do Brasil, números apontam que com tal fato, há uma superlotação dessa população “específica” o que faz com que isso, dentro da área da saúde, gere e resulte não só numa sobrecarga do sistema, mas no aumento da condição de risco e vulnerabilidade em que estão submetidos. (Santos, Cardoso, Brêda \& Costa,2015)

Diante de uma reflexão e apesar de todos os problemas como a superlotação nas celas, a precariedade e mediante a todas as dificuldades existentes acaba tornando esses locais em um ambiente bastante favorável ao desenvolvimento de agravos e doenças a saúde como também a falta de higiene, o sedentarismo, o uso de drogas, a precária assistência médica e a violência que rodeia essa área onde essa também favorece para os problemas de saúde dos indivíduos desse local. (Santos, et al., 2015)

No que se diz respeito a Constituição Federal (CF), que cita que a "saúde é um direito de todos e um dever do Estado", o Ministério da Saúde em ação integrada com o Ministério da Justiça elaborou o Plano Nacional de Saúde no Sistema Penitenciário (PNSSP) através da Portaria Interministerial $n^{\circ} 1.777$, de 09 de setembro de 2003 que prevê a inclusão da população penitenciária no SUS, garantindo que o direito à cidadania se efetive na perspectiva dos direitos humanos, como também que se criem estruturas de unidades básicas de saúde nos estabelecimentos prisionais. (Santos, et al., 2015)

Logo, podemos dizer também que ainda existem inúmeros impedimentos que fazem com que a prática e a conduta de saúde ao ser feita pelo enfermeiro, faz com que isso se torne bastante fragilizado em relação a ações quando se trata da humanização.

Com isso, é de extrema importância realizar a abordagem e a descrição da produção de conhecimento do profissional de enfermagem no contexto prisional, tendo em vista que o sistema carcerário possui grandes considerações a serem levadas em conta visando que há em si suas particularidades e especificidades como a carência e as dificuldades que nela se encontram para se fazer a humanização da assistência adequada. (Souza \& Passos, 2008) 
A motivação da pesquisa teve início através do interesse de uma Acadêmica de Enfermagem do Décimo Período, da graduação de enfermagem, da universidade Estácio de Sá, com intuito de analisar e pesquisar como o enfermeiro gestor atuante no sistema carcerário coloca em prática o conhecimento, as habilidades e as atitudes necessárias para se realizar a humanização da assistência de enfermagem e as dificuldades encontradas no mesmo.

A situação problema deste trabalho está relacionado ao devido fato que, apesar de se ter uma Portaria Interministerial e um Plano Nacional de Saúde do Sistema Penitenciário (PNSSP) onde busca e procura a assistência à saúde como um direito garantido às pessoas privadas de liberdade temos que levar em conta alguns fatores como: se fazer a humanização, prestar o cuidado de enfermagem com excelência e colocar o conhecimento e suas habilidades.

Diante disso, podemos então refletir que se fazer a humanização nesses locais tem se tornando um grande desafio para com essas pessoas fazendo assim, com que o profissional de enfermagem sinta uma "certa" necessidade em seu papel no contexto prisional, sendo este um dos profissionais capazes de promover mudanças nesse cenário contribuindo assim diretamente no melhoramento da evolução, do enriquecimento e do crescimento da saúde dessas pessoas privadas de liberdade.

A questão norteadora que irá direcionar a pesquisa: Como o enfermeiro atuante no sistema prisional pode exerce as práticas assistenciais para as pessoas Privadas de Liberdade e quais os principais obstáculos para a implementação da humanização do cuidado de enfermagem nesse âmbito?

$\mathrm{Na}$ tentativa de responder essa pergunta, surgiram os seguintes objetivos: analisar o significado do cuidado humanizado na assistência de enfermagem em um sistema prisional, identificar e discutir os principais obstáculos para a implementação desse cuidado estabelecendo estratégias para a implementação do conhecimento e suas práticas.

Essa pesquisa é fruto do interesse surgido de uma acadêmica do $10^{\circ}$ período da graduação em Enfermagem da Universidade Estácio de Sá, durante a atuação em um Núcleo de pesquisa (NUPENIG), onde foi mencionada em uma reunião a temática sobre o assunto. A partir disso, nota-se a necessidade da construção de um trabalho que pudesse inserir a temática em um setor onde a atuação do enfermeiro no sistema prisional é de difícil enfrentamento.

O objetivo é mencionar a atuação do enfermeiro no Sistema Prisional. O presente estudo tem como objetivos específicos identificar como o profissional lida e como é a assistência se tratando dessa população como também as estratégias e capacitações utilizadas para se promover o cuidado.

Esta pesquisa se justifica, pois, com os saberes descritos e analisados, será possível promover melhor desempenho dos profissionais tendo em vista que, no Brasil, a assistência à saúde á pessoas privada liberdade é classificada por carência e com isso, nota-se a necessidade de mudanças.

Diante disso, quando feita a pesquisa, observou-se que o número de procura e de estudos sobre esse tema em saúde penitenciária mostra-se insipiente na literatura nacional, mesmo em face do crescente interesse da graduação em enfermagem em pesquisar e analisar sobre o assunto.

Este estudo possui concordância de caráter social e acadêmica, pois cita diretamente a atuação do enfermeiro englobando os cuidados com pessoas privativas de liberdade. Sendo capaz de fornecer conhecimento através de lacunas para a humanização do cuidado prestado.

\section{Metodologia}

Trata-se de um estudo de abordagem qualitativa, do tipo revisão integrativa, abordando a temática: o enfermeiro gestor atuante no sistema carcerário: habilidades, conhecimentos e atitudes necessárias para se fazer a humanização da assistência. A revisão do processo baseou-se nas recomendações da lista de conferência preferred reporting items for systematic reviews and meta-analyses (PRISMA). 
Para a construção do artigo abordou-se as seis etapas que são: a identificação do tema e seleção da hipótese, estabelecimento da estratégia de pesquisa, definição e coleta de dados, análise dos dados coletados, interpretação e apresentação dos resultados, onde será abordada a humanização na Unidade de Terapia Intensiva Adulto. A revisão do processo baseou-se nas recomendações da lista de conferência Preferred Reporting Items for Systematic Reviews and MetaAnalyses. (PRISMA)

Figura 1 - Fluxograma de informação com as diferentes fases de uma revisão sistemática.

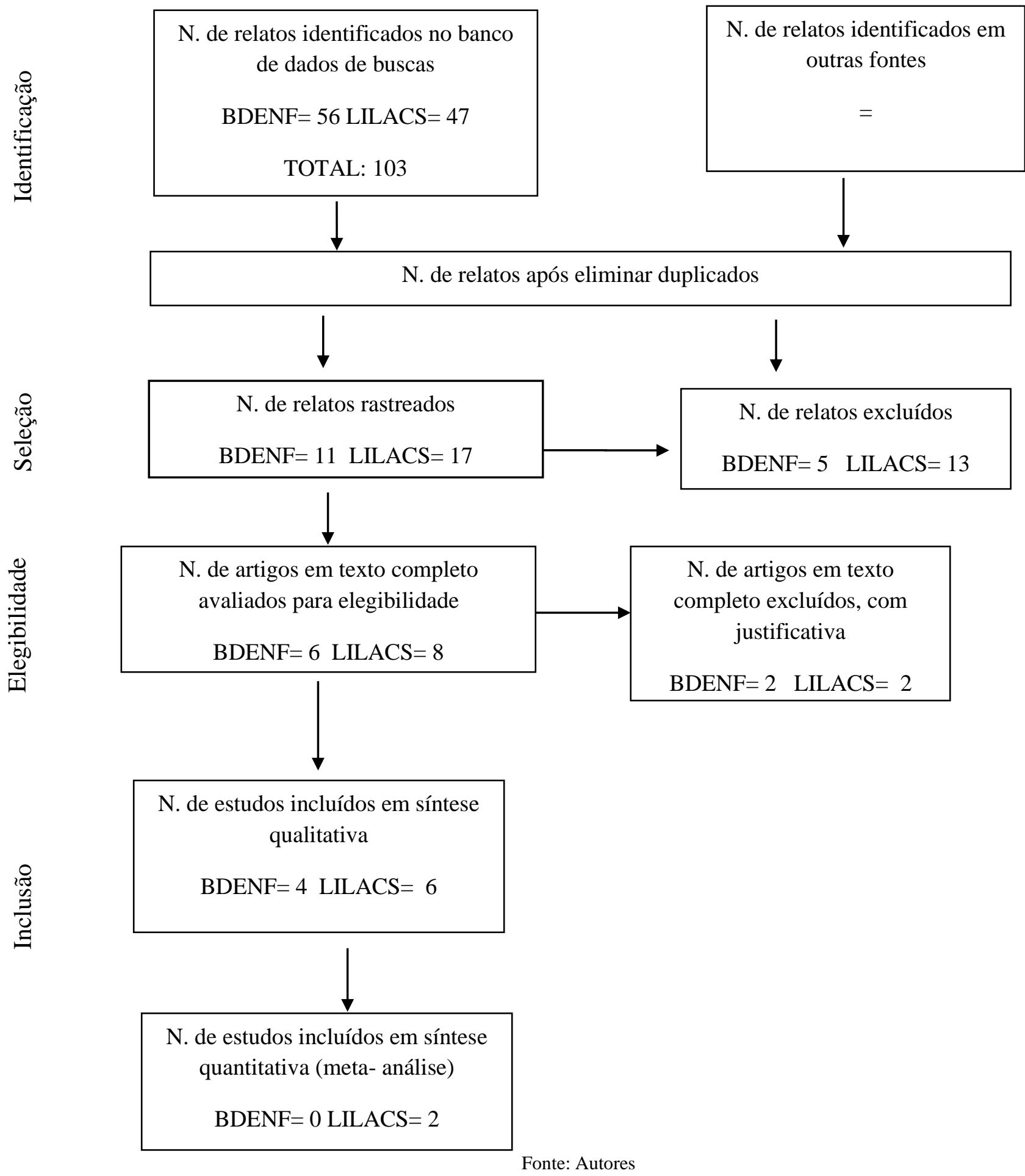


A questão norteadora foi construída de forma clara e específica, acoplada a um raciocínio teórico, adicionando teorias e entendimentos já absorvidos pelos discentes. Desta forma explorará publicações cientificas sobre o enfermeiro gestor atuante no sistema carcerário: habilidades, conhecimentos e atitudes necessárias para se fazer a humanização da assistência

A pergunta norteadora que irá direcionar a pesquisa: Como é a atuação do enfermeiro no Sistema Prisional? Na tentativa de responder a mesma, surgiu o seguinte objetivo: Identificar as habilidades, os conhecimentos e as atitudes de estratégias em prol da aplicabilidade do cuidado humanizado de Enfermagem dentro do sistema prisional.

Diante disso, a temática desse trabalho é baseada na estratégia PICO onde coloca a prática em evidência que sugere que as dificuldades apresentadas no ensino, pesquisa ou na prática assistencial sejam transformados e em seguida organizados que é definida como o acrônimo para Paciente, Problema ou População, Intervenção e Contexto.

Quadro 1 - Componentes da pergunta de pesquisa, seguindo-se o anagrama de PICO.

\begin{tabular}{|c|c|c|}
\hline Acrônimo & Descrição & Componentes da questão \\
\hline $\mathrm{P}$ & População & Enfermagem \\
\hline $\mathrm{I}$ & Intervenção & Prestação de Cuidados a Saúde \\
\hline $\mathrm{CO}$ & Contexto & Sistema Prisional \\
\hline
\end{tabular}

Fonte: Autores.

A estratégia de identificação e seleção dos artigos originou-se através da busca da Biblioteca Virtual de Saúde (BVS), com acesso as seguintes bases de dados eletrônicas: a Literatura Latino-Americana e do Caribe em Ciências da Saúde (LILACS) e Banco de Dados em Enfermagem (BDENF). A busca pelos periódicos teve início no mês de agosto de 2020, os materiais que contivessem em seu resumo os descritores em ciências da saúde (DeCS): saúde and enfermagem and prisões e com o uso do boleador "and". Foram adotados os seguintes critérios de inclusão para seleção dos artigos: materiais completos, disponíveis, em língua portuguesa, inglesa e espanhola entre 2016 a 2021. Os critérios de exclusão foram os artigos duplicados, incompletos e que não atendiam a proposta do presente estudo.

A pré-seleção de artigos foi feita pela leitura preliminar de títulos e resumos. Os estudos pré-selecionados foram lidos na íntegra para seleção final dos artigos para análise. Esta fase está representada na Figura 1.

Em relação aos dez artigos selecionados, foram tabelados individualmente, por título, autores, ano de publicação, periódico, objetivos, métodos, nível de evidência e considerações finais. A partir de então iniciou a análise bibliométrica destes que foram agrupados por similaridade sob forma de categorias. 
Figura 2. Fluxograma da seleção de estudos sobre a Humanização e UTI Adulto entre 2016 a 2021.

Número de artigos encontrados com base nas buscas realizadas na BVS

Descritores: Humanização da Assistência AND Cuidados AND Enfermagem

LILACS: 47

BDENF: 56

MEDLINE: 350

TOTAL: 426

Número de estudos rastreados publicados nos últimos 10 anos (2011-2021), textos íntegros em língua portuguesa, inglesa e espanhola.

LILACS: 42

BDENF: 53

MEDLINE: 64

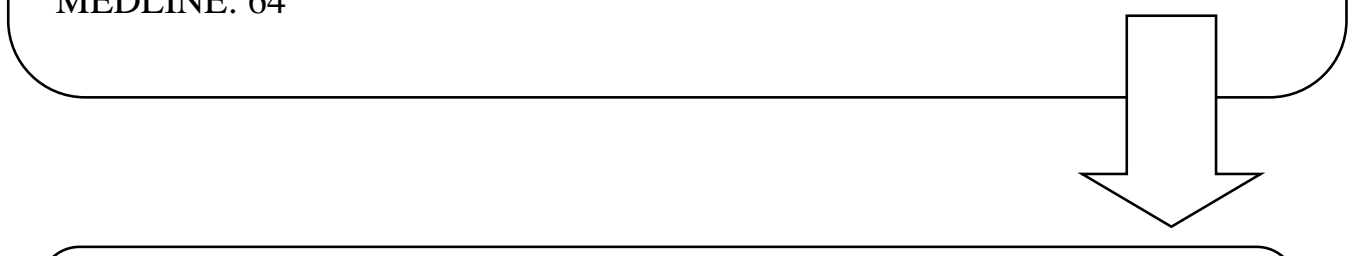

Exclusão: Realizada após a leitura do resumo dos artigos que não atendiam a temática e artigos duplicados.

\section{$\mathrm{N}^{\circ}$ de estudos excluídos: 114}

$\mathrm{N}^{\mathrm{o}}$ de artigos em texto completo a serem utilizados na presente pesquisa

LILACS: 7

BDENF: 3

TOTAL: 10

Fonte: Autores.

\section{Resultados e Discussão}

Assim, emergiram 11 artigos nas bases de dados pesquisadas através das estratégias de busca (figura 1) com os critérios citados anteriormente, os quais procederam-se à leitura minuciosa para a construção do conteúdo, destacando aqueles 
que responderam ao objetivo proposto pelo estudo, com intuito de organizar os dados de acordo com o objetivo proposto.

Os resultados apresentados no estudo mostram como o enfermeiro gestor atua no sistema carcerário de acordo com suas habilidades, conhecimentos e atitudes necessárias para se fazer a humanização da assistência nesse ambiente, sendo descritos no Quadro 1.

Em relação as características dos artigos foram visualizadas que depois a leitura do quadro falando sobre a questão dos métodos são todos de metodologia qualitativa, sendo 7 deles na base de dados da LILACS e 3 artigos da BDENF e 1 da SCIELO.

Quadro 2: Características dos artigos analisados no período de 2016 a 2020.

\begin{tabular}{|c|c|c|c|c|c|}
\hline Título & Autoria & Ano & Objetivo & Métodos & Considerações Finais \\
\hline $\begin{array}{l}\text { Enfermería penitenciaria: } \\
\text { marco legal y realidad } \\
\text { asistencial / Prison nursing: } \\
\text { legal framework and care } \\
\text { reality }\end{array}$ & Carrasco-Baún, H; s.af & 2017 & $\begin{array}{l}\text { explorar a realidade do bem- } \\
\text { estar da Enfermagem Prisional } \\
\text { coletando e analisando os } \\
\text { principais documentos legais } \\
\text { que regulam as prisões nas } \\
\text { prisões espanholas. }\end{array}$ & $\begin{array}{c}\text { Pesquisa } \\
\text { qualitativa- } \\
\text { descritiva. }\end{array}$ & $\begin{array}{l}\text { A necessária integração } \\
\text { da Saúde Prisional ao } \\
\text { Sistema de Saúde deve } \\
\text { ser repensada de forma a } \\
\text { garantir a igualdade e } \\
\text { cuidados de saúde para } \\
\text { pessoas privadas de } \\
\text { liberdade. }\end{array}$ \\
\hline $\begin{array}{l}\text { Saúde no Sistema } \\
\text { Penitenciário: o que falam } \\
\text { os trabalhadores de } \\
\text { enfermagem / Salud en las } \\
\text { Prisiones: lo que hablan los } \\
\text { trabajadores de enfermería / } \\
\text { Health in Prisons: the } \\
\text { speaking nursing workers }\end{array}$ & $\begin{array}{l}\text { Dos Santos, Fernanda } \\
\text { de Jesus; Cardoso, } \\
\text { Danielly Santos Dos } \\
\text { Anjos; Brêda, Mércia } \\
\text { Zeviani; Costa, Laís de } \\
\text { Miranda Crispim. }\end{array}$ & 2015 & $\begin{array}{l}\text { analisar } \\
\text { a atenção em saúde prestada } \\
\text { às pessoas em regime de } \\
\text { reclusão no sistema } \\
\text { penitenciário } \\
\text { na visão dos trabalhadores de e } \\
\text { nfermagem. }\end{array}$ & $\begin{array}{l}\text { Estudo } \\
\text { exploratório, } \\
\text { de abordagem } \\
\text { qualitativa }\end{array}$ & $\begin{array}{l}\text { Contribui para } \\
\text { a construção do conheciment } \\
\text { o sobre a temática em } \\
\text { questão, e a reflexão sobre } \\
\text { a atenção em saúde ofertada } \\
\text { no Sistema Penitenciário }\end{array}$ \\
\hline $\begin{array}{l}\text { Atenção básica à saúde de } \\
\text { apenados no sistema } \\
\text { penitenciário: subsídios } \\
\text { para a atuação da } \\
\text { enfermagem / Primary } \\
\text { health care of convicts in } \\
\text { the penitentiary system: } \\
\text { subsides for nursing } \\
\text { performance }\end{array}$ & $\begin{array}{l}\text { Barbosa, } \\
\text { Lima; Celino, Suely } \\
\text { Deysny } \\
\text { Matos; Oliveira, } \\
\text { Lannuzya Veríssimo } \\
\text { e; Pedraza, Dixis } \\
\text { Figueroa; Costa, } \\
\text { Gabriela } \\
\text { Cavalcanti. }\end{array}$ & 2014 & $\begin{array}{l}\text { Descrever aspectos estruturais e } \\
\text { características } \\
\text { do trabalho na atenção básica à } \\
\text { saúde em penitenciárias mascul } \\
\text { inas e fornecer subsídios para a } \\
\text { atuação do enfermeiro nesse } \\
\text { cenário. }\end{array}$ & $\begin{array}{l}\text { Estudo } \\
\text { descritivo e } \\
\text { quantitativo }\end{array}$ & $\begin{array}{l}\text { Nota-se que a implantação } \\
\text { do PNSSP parece apontar o } \\
\text { fim de } \quad \text { uma série de } \\
\text { paradigmas } \quad \text { relacionados } \\
\text { à saúde dos apenados.... }\end{array}$ \\
\hline $\begin{array}{l}\text { The Relationship Between } \\
\text { the Quality of Work and } \\
\text { Organizational } \\
\text { Commitment of Prison } \\
\text { Nurses. }\end{array}$ & $\begin{array}{l}\text { Karaaslan, } \\
\text { Ayfer; Aslan, Manar. }\end{array}$ & 2019 & $\begin{array}{l}\text { avaliar a qualidade de vida } \\
\text { relacionada ao trabalho e o } \\
\text { comprometimento } \\
\text { organizacional dos enfermeiros } \\
\text { que trabalham em presídios e } \\
\text { centros de detenção. }\end{array}$ & $\begin{array}{l}\text { Estudo } \\
\text { qualitativo, } \\
\text { quantitativo }\end{array}$ & $\begin{array}{l}\text { Os administradores devem } \\
\text { garantir a qualidade de vida } \\
\text { dos enfermeiros prisionais, } \\
\text { entendendo as dificuldades } \\
\text { da enfermagem prisional. }\end{array}$ \\
\hline $\begin{array}{l}\text { Nursing actions for liberty } \\
\text { deprived people: a scoping } \\
\text { review / Acciones de } \\
\text { enfermería para las } \\
\text { personas privadas de } \\
\text { libertad: una scoping review } \\
\text { / Ações de enfermagem } \\
\text { para as pessoas privadas de } \\
\text { liberdade: uma scoping } \\
\text { review }\end{array}$ & $\begin{array}{l}\text { Barbosa, Mayara } \\
\text { Lima; Medeiros, } \\
\text { Suzane Gomes } \\
\text { de; Chiavone, Flávia } \\
\text { Barreto } \\
\text { Tavares; Atanásio, } \\
\text { Lhana Lorena de } \\
\text { Melo; Costa, Gabriela } \\
\text { Maria } \\
\text { Cavalcanti; Santos, } \\
\text { Viviane Euzébia } \\
\text { Pereira }\end{array}$ & 2019 & $\begin{array}{l}\text { Identificar e mapear as práticas } \\
\text { assistenciais } \\
\text { pela equipe } \\
\text { enfermagem para as Pessoas } \\
\text { Privadas de Liberdade. }\end{array}$ & $\begin{array}{l}\text { Estudo } \\
\text { descritivo. }\end{array}$ & $\begin{array}{l}\text { Informa sobre as ações } \\
\text { desempenhadas e contribui } \\
\text { para a formação } \\
\text { de enfermeiros para } \\
\text { atuação no sistema prisional. }\end{array}$ \\
\hline
\end{tabular}




\begin{tabular}{|c|c|c|c|c|c|}
\hline $\begin{array}{l}\text { Sistematização da } \\
\text { assistência de enfermagem } \\
\text { em uma unidadeprisional } \\
\text { fundamentada na teoria de } \\
\text { orem / Nursing assistance } \\
\text { systematization in a prison } \\
\text { unit underpinned by orem } \\
\text { theory }\end{array}$ & $\begin{array}{lr}\text { Silva, Andréia } & \text { Alves de } \\
\text { Sena; Sousa, } & \text { Karinna } \\
\text { Alves } & \text { Amorim } \\
\text { de; Araújo, } & \text { Telma } \\
\text { Maria Evangelista de. . }\end{array}$ & 2017 & $\begin{array}{l}\text { relatar a experiência da prática } \\
\text { da sistematização da assistência } \\
\text { de enfermagem, com base nas } \\
\text { demandas terapêuticas } \\
\text { de autocuidado de acordo com } \\
\text { a teoria de orem, } \\
\text { para assistência à saúde em } \\
\text { uma Unidade Prisional. }\end{array}$ & $\begin{array}{l}\text { Abordagem } \\
\text { quantitativa. }\end{array}$ & $\begin{array}{l}\text { compreender a realidade } \\
\text { vivenciada pelo enfermeiro } \\
\text { no ambiente prisional e } \\
\text { refletir sobre a importância } \\
\text { da implantação da } \\
\text { sistematização da assistência }\end{array}$ \\
\hline $\begin{array}{l}\text { Percepção da equipe de } \\
\text { enfermagem acerca da } \\
\text { assistência à saúde no } \\
\text { sistema prisional / } \\
\text { Perception of Tthe nursing } \\
\text { team about health care in } \\
\text { the prison system / } \\
\text { Percepción del equipo de } \\
\text { enfermería acerca de la } \\
\text { asistencia a la salud en el } \\
\text { sistema prisional }\end{array}$ & $\begin{array}{l}\text { Santana, Júlio Cesar } \\
\text { Batista; Andrade, } \\
\text { Fernanda Cristina de }\end{array}$ & 2019 & $\begin{array}{l}\text { Compreender como a equipe de } \\
\text { enfermagem percebe } \\
\text { assistência da saúde no Sistema } \\
\text { Prisional. }\end{array}$ & $\begin{array}{l}\text { Estudo } \\
\text { qualitativo. }\end{array}$ & $\begin{array}{l}\text { é importante que haja um } \\
\text { processo } \\
\text { de organização administrativ } \\
\text { a básica, para melhorar os } \\
\text { recursos organizacionais e } \\
\text { capacitar os profissionais. }\end{array}$ \\
\hline $\begin{array}{lr}\text { Produção científica sobre } \\
\text { saúde penitenciária na } \\
\text { enfermagem brasileira / } \\
\text { Producción científica en } \\
\text { salud penitenciaria en } \\
\text { enfermería brasileña / } \\
\text { Scientific production on } \\
\text { penitentiary health in } \\
\text { Brazilian nursing }\end{array}$ & $\begin{array}{l}\text { Barbosa, Mayara } \\
\text { Lima; Neto, Alcides } \\
\text { Viana de } \\
\text { Lima; Saraiva, Cecília } \\
\text { Olívia Paraguai de } \\
\text { Oliveira; Bezerril, } \\
\text { Manacés dos } \\
\text { Santos; Costa, Gabriela } \\
\text { Maria } \\
\text { Cavalcanti; Santos, } \\
\text { Viviane Euzébia } \\
\text { Pereira. }\end{array}$ & 2019 & $\begin{array}{l}\text { Pesquisa em Enfermagem que } \\
\text { abordam a } \\
\text { temática saúde penitenciária. }\end{array}$ & $\begin{array}{l}\text { Pesquisa } \\
\text { qualitativa. }\end{array}$ & $\begin{array}{l}\text { Fomentar a discussão sobre } \\
\text { o tema representa um } \\
\text { avanço e é essencial para } \\
\text { suprir as lacunas verificadas } \\
\text { na prestação da assistência à } \\
\text { saúde nesse cenário. }\end{array}$ \\
\hline $\begin{array}{l}\text { A saúde dos homens } \\
\text { privados de liberdade no } \\
\text { Brasil / The health of } \\
\text { private freedom men in } \\
\text { Brazil / La salud de los } \\
\text { hombres de libertad privada } \\
\text { em Brasil }\end{array}$ & $\begin{array}{lr}\text { Cristo, Micheli; Diniz, } \\
\text { Michele } \\
\text { Lopes; Conceição, } \\
\text { Vander } \\
\text { da; Léo, } \\
\text { Martins } & \text { Marcela } \\
\text { de; Santos, } & \text { Furlan } \\
\text { Araujo doserson } \\
\text { Rafaela Simoneti, } \\
\text { Abrantes de Oliveira. }\end{array}$ & 2020 & $\begin{array}{l}\text { analisar as produções } \\
\text { científicas } \\
\text { a respeito do cuidado em saúde } \\
\text { dos homens no contexto } \\
\text { prisional. }\end{array}$ & $\begin{array}{l}\text { Pesquisa } \\
\text { qualitativa } \\
\text { descritiva. }\end{array}$ & $\begin{array}{l}\text { Os profissionais de saúde e } \\
\text { agentes de segurança atuam } \\
\text { em um simples } \\
\text { assistencialismo, } \\
\text { sem humanização, } \\
\text { sem medidas preventivas } \\
\text { de saúde. }\end{array}$ \\
\hline $\begin{array}{l}\text { Vivências da equipe de } \\
\text { enfermagem no cotidiano } \\
\text { do sistema penal / } \\
\text { Experiencias del equipo de } \\
\text { enfermería en la vida } \\
\text { cotidiana del sistema de } \\
\text { justicia penal / Experiences } \\
\text { of the nursing team in the } \\
\text { routine of the correctional } \\
\text { system }\end{array}$ & $\begin{array}{l}\text { Soares, Ana Amélia } \\
\text { Melo; Castro, Gabriela } \\
\text { Miranda de } \\
\text { Oliveira; Almeida, } \\
\text { Isabelle Elias Monteiro } \\
\text { de; Monteiro, Luciana } \\
\text { Alves Silveira; Torres, } \\
\text { Lilian Machado }\end{array}$ & 2020 & $\begin{array}{l}\text { compreender o cotidiano vivido } \\
\text { pela equipe de Enfermagem no } \\
\text { sistema penal. }\end{array}$ & $\begin{array}{l}\text { Estudo } \\
\text { qualitativo }\end{array}$ & $\begin{array}{l}\text { o ambiente prisional não } \\
\text { favorece ações concretas } \\
\text { de promoção r da } \\
\text { saúde e prevenção de } \\
\text { agravos. }\end{array}$ \\
\hline $\begin{array}{l}\text { A prática de enfermagem no } \\
\text { sistema penal: limites e } \\
\text { possibilidades }\end{array}$ & $\begin{array}{l}\text { Mônica Oliveira da } \\
\text { Silva e Souza }{ }^{\mathrm{I}} \text { Joanir } \\
\text { Pereita Passos }\end{array}$ & 2008 & $\begin{array}{lll}\text { identificar os princípios } & \text { que } \\
\text { norteiam a prática } & \text { de } \\
\text { enfermagem e discutir } & \text { os } \\
\text { limites e as possibilidades } & \text { da } \\
\text { atuação da equipe } & \text { de } \\
\text { enfermagem nos serviços de } & \\
\text { saúde do Sistema Penal } & \end{array}$ & $\begin{array}{l}\text { Estudo } \\
\text { descritivo } \\
\text { qualitativo }\end{array}$ & $\begin{array}{l}\text { a prática do cuidado e a } \\
\text { relação de ajuda são os } \\
\text { princípios que norteiam a } \\
\text { atuação da enfermagem na } \\
\text { Penitenciária }\end{array}$ \\
\hline
\end{tabular}


Após análise dos artigos elencados neste estudo foram construídas as seguintes categorias, de acordo com a temática de humanização na UTI Adulto:

\section{Categoria 1: A Política Nacional de Atenção Integral à Saúde das Pessoas Privadas de Liberdade no Sistema Prisional (PNAISP), O Plano Nacional (PNSSP) e a Inserção do Enfermeiro no Âmbito Penitenciário}

No âmbito no que se refere à assistência à saúde as pessoas privativas de liberdade, foram criadas duas políticas de saúde específicas com um objetivo de fornecer tais necessidades para esta população. A primeira é o Plano Nacional de Saúde no Sistema Penitenciário (PNSSP), que se diz respeito a garantia do acesso ao Sistema Único de Saúde (SUS), onde ela se refere a atenção à saúde integral e universal para todos aqueles que pertencem à população encarcerada em regime fechado $\mathrm{e}$ de ambos os sexos, seja feminino ou masculino. (Barbosa, Celino, Oliveira, Pedraza, \& Costa, 2014)

A Organização dos serviços de saúde no sistema penitenciário foi construída pela Portaria no 1.777 de 09 de setembro de 2003, com o Plano Nacional de Saúde no Sistema Penitenciário (PNSSP).Ela se diz a respeito da assistência à saúde das pessoas privadas de liberdade, com foco nos princípios e diretrizes do Sistema único de Saúde (SUS), abordando que o direito à cidadania se dá a todos possibilitando o acesso a ações e serviços de saúde igualitários .Com base nisso, a assistência à saúde dentro desse ambiente é feita de maneira no que se diz a respeito da atenção primária, onde há a prevenção das doenças, a vigilância e a promoção da saúde . A PNSSP determina vários critérios, como por exemplo, a inclusão do enfermeiro na equipe onde o mesmo é responsável na atuação da assistência à população prisional. (Silva, Souza \& Araújo ,2017)

Diante disso, a colocação de profissionais de saúde no meio penitenciário aconteceu desde a PNSSP e continuou após a PNAISP. Em concordância com a política, a acessibilidade e a atenção à saúde são desenvolvidas e amplificadas pelas Equipes de Atenção Básica no Sistema Prisional (EABP). Barbosa et al. (2019)

Para que seja implementada a PNSSP, sabemos que isso depende de uma contribuição na qual, conforme as diretrizes de saúde é importante a inclusão de profissionais como médicos, assistentes sociais, psicólogo, odontólogo, o enfermeiro e o técnico de enfermagem, atentando que sem essa organização não há como ter o controle ou a redução dos agravos à saúde para a população penitenciária brasileira (Barbosa et al., 2014)

Tendo em vista que a PNSSP promove o controle de tuberculose, hipertensão, diabetes ,hanseníase, diagnóstico, aconselhamento e tratamento contra as IST/HIV/AIDS e a atenção em saúde mental, para se desenvolver essas ações, os profissionais de enfermagem tem um papel mega importante, visando que os mesmos, são integrantes da equipe de atenção básica, onde desenvolvem atividades tanto no individual quanto no meio coletivo, fazendo assim a promoção da saúde e prevenção de doenças. (Barbosa et al., 2014)

A enfermagem então, como responsável pela prática do cuidado, e tratando de um olhar totalmente holístico quando se tratar de saúde, deve assim, promover no ambiente de saúde um conjunto de ações que supram as necessidades de cada população fazendo assim que o profissional enfermeiro desenvolva suas atividades pautadas no que diz respeito em cada lei seja ela: o exercício profissional, a política da atenção básica à saúde ou nos princípios da ética. A resolução no 358/2009 do Conselho Federal de Enfermagem (COFEN), diz que a assistência de enfermagem, deve então ser sistematizada por meio da execução e realização do processo de enfermagem e registrada em prontuário, realizada em toda e qualquer instituição de saúde. Ele então é mantido por uma teoria de enfermagem que qualifica os cuidados prestados, humaniza o atendimento, define o papel do enfermeiro e assim dá autonomia à profissão. (Silva et al., 2017)

\section{Categoria 2: O Papel da Enfermagem no Sistema Prisional}

A história da Enfermagem Penitenciária Espanhola do século XVI e XVII foi de grande importância para se fazer a enfermagem moderna nos dias de hoje. Ela aborda que a verdadeira função da enfermagem é constituir sua função no âmbito 
do ensino, do atendimento, da gestão e da pesquisa, ou seja, o tratamento e a cura de uma pessoa doente estavam entrelaçados ao físico, a saúde mental e o social desse indivíduo. (Baún, 2017)

Para se fazer as ações de saúde, sabemos que na enfermagem, é fundamental e de grande importância a criação de uma boa estrutura física em sua efetividade. Com isso, tendo em vista que as condições insalubres reconhecidas dentro dos ambientes prisionais não é uma condição favorável como a alimentação de má qualidade, estrutura física inadequada e outras coisas mais, acabam gerando situações de vulnerabilidade para a enfermagem atuar em sua devida profissão e assim mantendo a relação de aparecimentos e agravos a saúde dos mesmos, sabendo que a falta de compreensão e de informação sobre o tema : saúde no geral , é de bastante precariedade . (Barbosa et al., 2014)

O enfermeiro no âmbito penitenciário tem um papel fundamental quanto as suas funções como por exemplo a atuação na saúde das PVL objetivamente na Atenção primária ou em outros setores. Há também a fundamental importância de destacar a assistência nas quais eles prestam a pacientes incluídos em programas como HIV, hepatite C, tuberculose, saúde mental e dependência de drogas, bem como para os cuidados de saúde para o pronto-socorro, que torna esse atendimento específico e diferente. Há também enfermeiros que atuam em Centros crônicos onde esse doente permanece por um longo período de tempo por terem alguma doença crônica. Com isso, podemos citar que além de todas essas funções, o enfermeiro além de promover saúde, ele tem o papel de pesquisador, professor e gestor dentro das enfermarias penitenciárias. (Santos, et al., 2015)

A enfermagem penitenciária então, se torna um serviço que oferece além da promoção de saúde , a educação, o exame físico, a distribuição de medicamentos, a adesão e continuidade do tratamento, os primeiros socorros, exames e cuidados pósoperatórios, além de atendimento médico de emergência como também a função de resolver problemas de saúde; fornecer orientação; coleta e registro de informações sobre o estado de saúde; realização de exames periódicos; administrar medicamentos prescritos pelo médico; determinar e acompanhar presidiários com problemas emocionais, doenças crônicas, doenças venéreas, desnutrição e maus hábitos como álcool, cigarro e uso de drogas; prevenção de acidentes e prestação de primeiros socorros. (Ayfer \& Manar,2019)

Nesse sentindo, podemos dizer que o trabalho dos profissionais de enfermagem deve ser capaz de alcançar as metas e ações propostas pelo PNSSP, garantindo assim, a assistência integral ao indivíduo inserido em penitenciárias. Nota-se também que, enquanto a segurança seja uma das principais prioridades nesse sistema, esse fato acaba gerando um empecilho para a assistência à saúde, fazendo assim que seja fundamental que o enfermeiro reconheça as especificidades do processo de trabalho nas penitenciárias, mantendo uma conduta ética e respeitando os princípios que regem a profissão, a fim de garantir o direito à saúde e dignidade humana. (Barbosa et al., 2014)

Assim, a enfermagem pode contribuir então para a libertação de uma condição de vida mais digna a essas pessoas, tanto no biológico, social e psicológico gerando o conforto e o bem estar, diminuindo assim, ações que de discriminação ou o preconceito, respeitando princípios éticos e legais, assim com o intuito de resgatar o sentido da existência humana. (Santos, et al., 2015)

\section{Categoria 3: Qualidade de Vida dos Profissionais Enfermeiros no Âmbito Prisional. Não Mexer em Nada}

O intuito do serviço prisional inclui conservar prisioneiros sob custódia e instituir um ambiente favorável, com cautela, disciplina e segurança na prisão; atendendo às necessidades básicas sendo um fator importante: a saúde. Com isso, podemos dizer que a enfermagem penitenciária possui um grande diferencial ao usarem todos os seus conhecimentos e habilidades diariamente no atendimento a esse doente pois além dos problemas de saúde, as enfermeiras tomam conhecimentos dos crimes, esperanças e desespero dos prisioneiros onde o fazem muitas das vezes se tornarem defensores de sua saúde, ou seja, esse ambiente (prisão) se torna um grande desafio em seu desenvolvimento profissional. Por isso, eles acabam se tornando diferenciados e precisam ser educados para serem assim, protegidos em tal âmbito. (Ayfer \& Manar, 2019). 
Os Enfermeiros que trabalham em prisões estão então expostos a vários problemas e fatores de segurança onde provavelmente atendem viciados em drogas ou álcool, aqueles que possuem personalidade agressiva, e também os que tem doenças crônicas e pode experimentar mudanças repentinas no estado de saúde mental. Diante desse fato, os enfermeiros que trabalham em prisões e nesses locais podem e tem problemas com a qualidade de vida relacionada ao seu trabalho nesse ambiente (Ayfer \& Manar,2019).

Podemos dizer também que as práticas de enfermagem no sistema penitenciário têm se envolvido na melhoria da assistência à saúde nesse ambiente, tendo em vista que o dinamismo se torna muito presente quando se tratado de um acesso e da capacidade de resolução relacionada aos serviços. Sendo assim, é de grande importância realizar parcerias entre o sistema prisional e outras organizações, e também rever o processo de trabalho da enfermagem se tratando do pós cuidado. Notando isso é de extrema importância que sejam feitos investimentos na formação de enfermeiros e demais profissionais de saúde para que assim, isso possa contribuir para a redução de agravos que ainda existem em relação ao tratamento da saúde mental no ambiente prisional seja dos mesmos e dos profissionais de saúde em si. (Barbosa et al.2019).

Isso se dá a uma reflexão necessária onde muitos profissionais de saúde se recusam a trabalhar nesses ambientes por causa de condições de trabalho precária onde isso acarreta no desenvolvimento profissional dentro da profissão que a solução seria que uma das possibilidades seria o incentivo à formação de profissionais de enfermagem com o perfil adequado à essa realidade. (Cristo et al.2020)

A vivência profissional nesse âmbito, tanto em relação a saúde quanto na segurança, acaba resultando em um trabalho com insatisfação, levando em consideração a deficiência de recursos materiais e descaso do poder público sucedendo em exposição aos riscos psicossociais, aborrecimentos e desgaste emocional dos trabalhadores de saúde ou seja ,muitos desses profissionais desenvolvem patologias psicológicas por se encontrarem desmotivados devido ao ambiente, a alta demanda de trabalho e a incompatibilidade com a dignidade humana profissional . (Santana \& Reis,2019).

Sendo assim, deve-se ter a compreensão, na perspectiva do profissional de Enfermagem que é atuante nesses setores, pois devemos analisar o vivido, a convivência e o cotidiano dos mesmos, permitindo assim, darmos uma maior visibilidade a essas experiências que qualificam a sua assistência e o seu diferencial desde a sua formação até a gestão do cuidado. (Soares, Castro, Almeida, Monteiro \& Torres, 2020).

\section{Conclusão}

Conclui-se que, a realidade vivenciada pelos profissionais de enfermagem que atuam nos serviços de saúde do Sistema Prisional, nos faz refletir que não basta estarmos com foco apenas nessas pessoas quando se pensa em realizar a humanização, mas, agir com princípios básicos à prática do cuidado e a relação de ajuda na atenção à saúde das pessoas privadas de liberdade. Realizando-se a prática do cuidado e a relação da ajuda com as ações da equipe de enfermagem, enfatizamos também que os profissionais de saúde, por seremos protagonistas do processo de cuidar, necessitam de reconhecimento e valorização do trabalho nesses setores. Muitas equipes sentem-se despreparadas para atuarem em setores como esses, e por isso, faz-se necessária a qualificação dos profissionais envolvidos. Com isso, esse estudo proporcionou as limitações e as possibilidades da equipe de enfermagem ao executar suas ações dentro desses setores. E, como possibilidades podemos destacar as mesmas habilidades e competências exercidas na realidade extramuros. Mediante a isso e , apesar dos limites que o Sistema Penitenciário impõe, conseguimos enxergar uma Enfermagem que desempenha suas atividades neste sistema assegurando o cumprimento éticos da profissão que são aqueles relacionados ao respeito à vida, a dignidade e os direitos da pessoa humana. 
Research, Society and Development, v. 10, n. 6, e34910615279, 2021

(CC BY 4.0) | ISSN 2525-3409 | DOI: http://dx.doi.org/10.33448/rsd-v10i6.15279

\section{Referências}

Santos, F. d. J. d., Cardoso, D. S. d. A., Brêda, M. Z., \& Costa, L. d. M. C. (2015). Saúde no Sistema Penitenciário: o que falam os trabalhadores de enfermagem, 41(19), 114-125. https://rua.ua.es/dspace/bitstream/10045/46619/3/Cultura-Cuidados_41_14.pdf

Souza, M. O. d. S. e., \& Passos, J. P. (2008). A prática de enfermagem no sistema penal: limites e possibilidades. Escola Anna Nery, 12(3). https://doi.org/https://dx.doi.org/10.1590/S1414-81452008000300004

Barbosa, M. L., Celino, S. D. d. M., Oliveira, L. V., Pedraza, D. F., \& Costa, G. M. C. (2014). Atenção básica à saúde de apenados no sistema penitenciário: subsídios para a atuação da enfermagem. Escola Anna Nery, 18(4). https://doi.org/10.5935/1414-8145.20140083

Silva, A. A. d. S., Souza, K. A. A. d., \& Araújo, T. M. E. (2017). Sistematização Da Assistência De Enfermagem Em Uma Unidade Prisional Fundamentada Na Teoria De Orem. Revista de Enfermagem da UFSM, 7(4), 725-735. https://doi.org/10.5902/2179769222076

Barbosa, M. L., Medeiros, S. G. d., Chiavone, F. B. T., Atanásio, L. L. d. M., Costa, G. M. C., \& Santos, V. E. P. (2019). Ações de enfermagem para pessoas privadas de liberdade: uma revisão do escopo. Escola Anna Nery, 23(3). https://doi.org///dx.doi.org/10.1590/2177-9465-ean-2019-0098

Baún, H. C. (2017). Enfermería penitenciaria: marco legal y realidad asistencial. Rev Esp Sanid Penit, 3(13), 8-18. https://scielo.isciii.es/pdf/sanipe/v19n1/es_02_especial.pdf

Ayfer, K., \& Manar, A. (2019). The Relationship Between the Quality of Work and Organizational Commitment of Prison Nurses. Journal of Nursing Research, 27(3), 25. https://doi.org/10.1097 / jnr.0000000000000286

Barbosa, M. L., Neto, A. V. d. L., Saraiva, C. O. P. d. O., Bezerril, M. d. S., Costa, G. M. C., \& Santos, V. E. P. (2019). Produção científica sobre saúde penitenciária na enfermagem brasileira. Rev. Enferm. UFSM - REUFSM, 9(70), 1-15. https://doi.org/10.5902/2179769232885

Cristo, M., Diniz, M. L., Conceição, V. M. d., Léo, M. M. F., Santos, J. A. d., \& Simoneti, R. A. A. d. O. (2020). A Saúde Dos Homens Privados De Liberdade No Brasil. Rev Fun Care Online. https://doi.org/http://dx.doi.org/10.9789/2175-5361. rpcfo.v12.8618

Santana, J. C. B., \& Reis, F. C. d. A. (2019). Percepção da Equipe de Enfermagem Acerca da Assistência à Saúde no Sistema Prisional. Rev Fund Care Online, 11(5). https://doi.org/10.9789/2175-5361.2019.v11i5.1142-1147

Soares, A. A. M., Castro, G. M. d. O., Almeida, I. E. M. d., Monteiro, L. A. S., \& Torres, L. M. (2020). Vivências Da Equipe De Enfermagem No Cotidiano Do Sistema Penal. Rev. baiana enferm, 34. https://doi.org/http://dx.doi.org/10.18471/rbe.v34.34815 